\title{
Monolayer-Functionalized CdTe Nanocrystals with Controlled Fluorescent Properties Based on Chemical Surface Modification
}

\author{
Takaaki Tsuruoka, Masanori Sugiyama, Rena Takahashi, Hidemi Nawafune, \\ and Kensuke Akamatsu \\ Frontiers of Innovative Research in Science and Technology (FIRST), Konan University, 7-1-20 \\ Minatojima-minamimachi, Chuo-ku, Kobe 650-0047, Japan
}

The significant enhancement of fluorescent quantum yields for CdTe nanocrystals (from 1 to $60 \%$ ) through chemical surface modifications is described, enabling us to synthesize monolayer-functionalized nanocrystals with controlled flurescent properties. Several detailed structural characterization for the purified nanocrystals upon reaction revealed that the process relies on the surface chemical attachment of thiolate ligands on the nanocrystal surface, probably with a defect site at the nanocrystal surface being compensated, allowing an increase in the probability of radiative recombination of excitons generated in the nanocrystals. The surface of the obtained nanocrystals could be further modified with other type of molecules through ligand exchange reaction without significant changes in structural and optical characteristics of the nanocrystals.

Key words: Semiconductor, Nanocrystals, Surface modification, Fluorescent enhancement

\section{INTRODUCTION}

Ligand-stabilized semiconductor nanocrystals are an important class of nanomaterials with unusual electrical and optical properties that are driven by quantum confinement effects [1]. Recently, such nanocrystals have been investigated for potential uses with light-emitting diodes (LEDs) [2], quantum dot lasers [3], and biological labels [4]. A primary requirement for the success of these applications is the ability to prepare highly luminescent nanocrystals. Typical synthetic strategies to construct such nanocrystals include alternative surface passivation of seed nanocrystals with inorganic materials, thus generating core-shell structures [5-10].

The surface passivation of nanocrystals with organic molecules, which yields ligand-stabilized nanocrystals, is key to obtaining stable nanocrystals that can be used as functional macromolecules for practical applications. One of the approaches to surface functionalization of nanocrystals is the exchange of stabilizing ligands with other molecules, which has been investigated in detail for metal nanoparticles. However, with the exception of CdTe nanocrystals $[11,12]$, the place exchange reactions for semiconductor nanocrystals has been reported to yield nanocrystals with difficult to control optical properties; reaction has often lead to a decrease in the fluorescent intensity of the nanocrystals [13,14]. Further, the fluorescence intensity of the "purified" semiconductor nanocrystals dispersed in solvents gradually decreases over a period of time due to the desorption of protective molecules [14]. Therefore, the synthesis of highly luminescent nanocrystals through surface functionalization of desired molecules with high affinity for nanocrystals has been of great interest. We recently demonstrated a novel fabrication method for highly luminescent thin films consisting of $\mathrm{CdTe}$ nanocrystals by immobilization of as-prepared nanocrytals followed by surface modification using thiol molecules [15]. Although we succeeded in improving the quantum yield of immobilized nanocrystals by compensating surface defects with organic ligands, the fully passivation of nanocrystal surface still remains to be achieved by an effect of steric hindrance.

Herein, we report a synthetic strategy to construct "highly luminescent" CdTe nanocrystals with a room temperature QY of ca. $60 \%$. This strategy relies on a chemical surface modification of the as-prepared nanocrystals (initial QY: below $1 \%$ ) with alkanethiols. The attachment of thiols to the CdTe surface is thought to effectively inhibit the nonradiative recombination process, resulting in changing optically "silent" nanocrystals to fluorescent nanocrystals. It is important to note that the process enables further surface modification of the nanocrystals with different ligands, yielding mixed monolayer-functionalized nanocrystals. Because the optical properties of the nanocrystals remain intact during the place exchange reaction, the process also allows the synthesis of highly luminescent nanocrystals with tailored surface chemistries, and therefore functionalities. 


\section{EXPERIMENTAL}

Synthesis of TBP/OPA-capped CdTe Nanocrystals.

Green- and red-emitting CdTe nanocrystals as starting materials were synthesized in noncoordinating solvents following the previous literature method [16]. Typically, CdO (0.1 mmol), $n$-octylphosphonic acid (OPA: 0.2 mmol), and octadecene (ODE: $15.6 \mathrm{mmol}$ ) were loaded into a $25 \mathrm{~mL}$ three-neck flask and heated to $260{ }^{\circ} \mathrm{C}$ under dry $\mathrm{Ar}$ flow to form clear solution. A mixture of $\mathrm{Te}(0.2$ mmol), tributylphosphine (TBP: $2.3 \mathrm{mmol}$ ), and ODE $(5.9 \mathrm{mmol})$ was injected into $\mathrm{Cd}$ precursor solution and then the resulting solution was kept at $260{ }^{\circ} \mathrm{C}$ to grow the CdTe nanocrystals. The mixture was cooled to room temperature when CdTe nanocrystals were achieved to desired nanocrystal size.

Surface Modification of Nanocrystals.

Alkanethiol molecules $(0.42 \mathrm{mmol})$ were added into toluene containing crude as-prepared CdTe nanocrystals (final concentration of nanocrystals: $13 \mathrm{nM}$ ), and then this mixture was kept at $80{ }^{\circ} \mathrm{C}$ for several hours. The reaction was terminated after appropriate time intervals by addition of mixture of methanol and acetone, and the resulting nanocrystals were separated using centrifugation followed by redispersion into toluene. After two more purification process, purified alkanethiol-capped CdTe nanocrsytals were obtained.

\section{Functionalization of Nanocrystals.}

THF-containing 11-mercapto-1-undecanol (MUO) or 11-mercaptoundecanoic acid (MUA) molecules was added into a THF solution of the purified DT-capped CdTe nanocrystals. After stirring for $3 \mathrm{~h}$, the nanocrystals were precipitated by addition of toluene and were separated using centrifugation. The resulting nanocrystals were redispersed into methanol and water ( $\mathrm{pH} 7.8$, adjusted using $\mathrm{NaOH}$ ) for DT/MUO-capped and DT/MUA-capped nanocrystals, respectively.

\section{RESULTS AND DISCUSSION}

\section{Synthesis and Fluorescent Enhancement.}

The present process for QY enhancement of CdTe nanocrystals involves the preparation of a solution containing starting CdTe nanocrystals, followed by addition of alkanethiol molecules. The as-prepared nanocrystals had both smaller (diameter: $2.7 \mathrm{~nm}$ ) and larger nanocrystals (diameter: $3.4 \mathrm{~nm}$ ), showing fluorescent emission wavelengths of $543 \mathrm{~nm}$ (green) and $615 \mathrm{~nm}$ (red), respectively. Since both nanocrystals dispersed in toluene showed fluorescent QYs of ca. $1 \%$, the initial dilute solution of the nanocrystals (concentration of the CdTe nanocrystals: $13 \mathrm{nM}$ ) appeared optically silent in fluorescence images (Figure 1). However, upon addition of 1-dodecanethiol (DDT) to a solution of the nanocrystals in toluene at $80{ }^{\circ} \mathrm{C}$, the nanocrystals gradually became fluorescent after a few hours, and the solution remained clear for both red- and
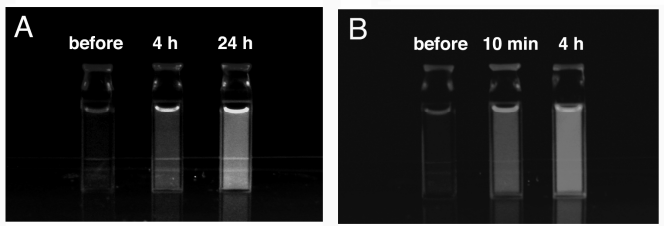

Figure 1. Fluorescent images of the toluene solution of (A) green- and (B) red-emitting CdTe nanocrystals before and after adding DDT. Concentration of the nanocrystals: $13 \mathrm{nM}$.
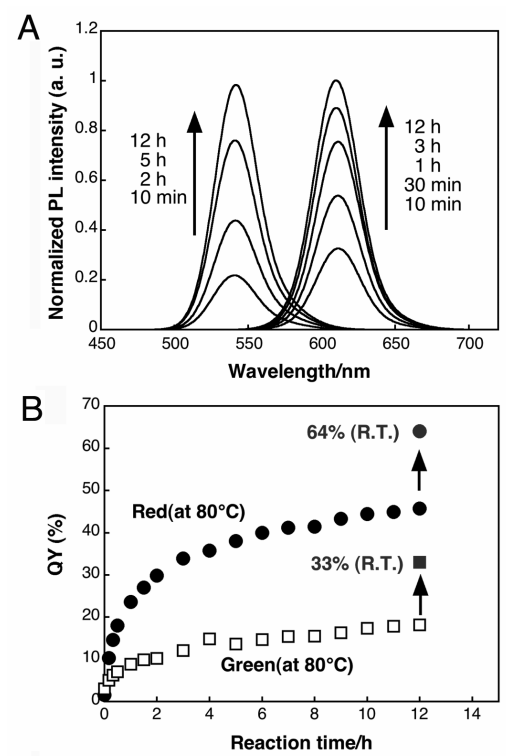

Figure 2. Changes in fluorescence spectra for green- and red-emitting CdTe nanocrystals in toluene as a function of time after adding DDT. (B) Changes in fluorescent QYs of the nanocrystal at $80^{\circ} \mathrm{C}$ as a function of time after adding DDT.

green-emitting nanocrystals. In time course of the emission spectra shown in Figure 2A, the photoluminescence (PL) intensity of CdTe nanocrystals was observed to significantly increase as the reaction time increased. The PL peak wavelength and full width at half maximum remained unchanged. The position of the absorption edge and the absorbance of CdTe nanocrystals remained unchanged during the reaction. These results indicate that the nanocrystal size remains intact, and there is no significant aggregation of nanocrystals upon addition of DDT.

Since the fluorescence measurement was performed for a solution containing nanocrystals at a fixed concentration (13 $\mathrm{nM})$, the observed changes in fluorescence essentially reflect the intrinsic fluorescent QYs of the CdTe nanocrystals. The QY of the nanocrystals was observed to increase remarkably at the initial stage of reaction and then become saturated, as shown in Figure 2B. This trend is similar to the changes 
Table 1. Atomic contents of purified CdTe nanocrystals obtained after various reaction times with DDT.

\begin{tabular}{ccccc}
\hline $\begin{array}{c}\text { Reaction time } \\
{[\mathrm{h}]}\end{array}$ & $\begin{array}{c}\mathrm{Cd} \\
{[\%]}\end{array}$ & $\begin{array}{c}\mathrm{Te} \\
{[\%]}\end{array}$ & $\begin{array}{c}\mathrm{P} \\
{[\%]}\end{array}$ & $\begin{array}{c}\mathrm{S} \\
{[\%]}\end{array}$ \\
\hline 0 & 48.1 & 44.0 & 7.9 & - \\
0.5 & 44.8 & 40.9 & 5.8 & 8.5 \\
10 & 43.7 & 41.6 & 5.1 & 9.6 \\
\hline
\end{tabular}
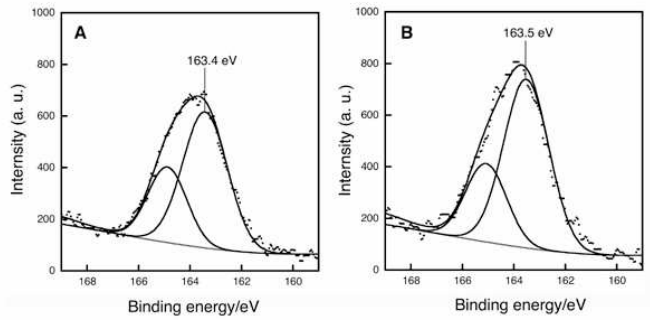

Figure 3. XPS data for the $\mathrm{S} 2 \mathrm{p}$ core levels of CdTe nanocrystals obtained after reaction for $30 \mathrm{~min}(\mathrm{~A})$ and $10 \mathrm{~h}(\mathrm{~B})$.

in integral intensity of the emission peak. For red-emitting nanocrystals, the $\mathrm{QY}$ at $80{ }^{\circ} \mathrm{C}$ is improved from an initial $1 \%$ up to $50 \%$ upon addition of DDT for $12 \mathrm{~h}$ (room temperature QY: $64 \%$ ). A similar improvement in QY is observed for the green-emitting nanocrystals ( $1 \%$ up to $18 \% 12 \mathrm{~h}$ after addition of DDT, room temperature QY: $33 \%$ ), indicating that the observed fluorescence enhancement effect is independent of nanocrystal size. It is important to note that the temperature and the concentration of DDT employed in this reaction had a remarkable effect on the degree of fluorescence enhancement. In general, a higher temperature and concentration of DDT (relative to the CdTe nanocrystals) gave a faster rate of increase in QYs. Thus, the fluorescence QYs can be controlled simply by altering these experimental conditions.

\section{Fluorescent Enhancement Process.}

This enhancement process is proposed to occur through a surface attachment of a thiolate, with a defect site at the nanocrystal surface being compensated, thereby increasing radiative pathways for exciton recombination process. Several observations are consistent with this mechanism. First, the EDX elemental analysis (Table 1) suggests that exchange with TBP/OPA and attachment of thiol molecules at the nanocrystal surface is only a change that occurs during reaction. In addition, the XPS analysis (Figure 3) clearly demonstrates that the presence of sulfurs in thiol molecules, indicating the nanocrystals consist of pure CdTe cores with organic shells. Since TBP and OPA molecules can only coordinates to $\mathrm{Te}, \mathrm{Cd}$ atoms on the nanocrystal surface are not effectively capped, providing effective thiol attachment process. The high affinity of the mercapto group for the $\mathrm{Cd}$ atoms is, therefore, responsible for increase in PL intensity during initial stage of reaction that make the highly defective nanocrystals to the less defective ones through chemical attachment to the $\mathrm{Cd}$ surface atoms. Interestingly, although the components of nanocrystals were almost the same after the reaction of $30 \mathrm{~min}$ and $10 \mathrm{~h}$, the QYs increased with increasing the reaction time. It is well-known that the QYs of semiconductor nanocrystals depend on a configuration of organic ligands [17]. In the present case, the high density of organic ligand on nanocrystal surface may induced the interaction between ligands such as van der Waals interaction, resulting in optimizing configuration. Second, an additional experiment in which the $n$-alkanes were added into the solution, instead of alkanethiols, did not result in fluorescent enhancement, thus demonstrating that the mercapto moiety is critical for affecting the fluorescent enhancement process. Finally, in the time course of the fluorescence decay curves for the nanocrystals in the surface modification reaction, the lifetime is observed to significantly increase upon reaction for an initial $1 \mathrm{~h}$, and then slightly increase as the reaction time increases (from $13 \mathrm{~ns}$ before reaction to $27 \mathrm{~ns} 4 \mathrm{~h}$ after reaction). These values are comparable to those reported in the literature [10]. These results demonstrate that the radiative recombination process becomes dominant upon the reaction because of the reduced concentration of surface defects as a consequence of the thiol capping. It is suggested to be this effect that contributes to the increased QYs.

\section{Surface Functionalization of Nanocrystals.}

The relative stability and easy handling nature of the resultant purified nanocrystals (as solid powders) is expected to enable subsequent introduction of other functional groups. In an effort to demonstrate this capability, a place-exchange reaction using 11-mercaptoundecanol (MUO) and 11-mercaptoundecanoic acid (MUA) for decanthiol (DT)-capped CdTe nanocrystals prepared by an analogous procedure to that described above (QY: $33 \%$, reaction time: $3 \mathrm{~h}$ ) was conducted. After appropriate reaction times, mixed monolayer-protected $\mathrm{CdTe}$ nanocrystals were obtained (DT/MUO and DT/MUA-capped nanocrystals), and the degree of introduction of thiolate bearing polar moieties was controlled simply by the concentration of molecules and the reaction time. Typical examples of both purified mixed monolayer-protected $\mathrm{CdTe}$ nanocrystals are soluble in polar solvents such as alcohol and DMF, and the DT/MUA-capped nanocrystals are also soluble in water (pH 7.8 adjusted using $\mathrm{NaOH}$ ). Previous study by 


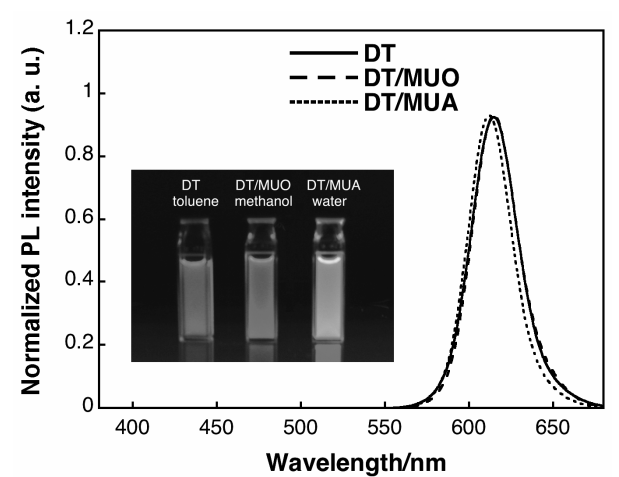

Figure 4. Fluorescence spectra of DT-functionalized CdTe nanocrystals in toluene, DT/MUO-functionalized nanocrystals in methanol, and DT/MUA-functionalized nanocrystals in water. (Inset) fluorescent images of these solutions containing the nanocrystals.

Gaponik et al. for ligand exchange of thioglicolic acid-stabilized CdTe nanocrystals with dodecanethiol (through direct phase transfer from water without phase-transfer reagents) resulted in partial decrease in QY [18]. The optical properties of the nanocrystals in the present study remain relatively unaltered after place exchange (Figure 4): emission wavelengths for these nanocrystals are nearly consistent with those for initial DT-capped nanocrystals, and the resulting nanocrystals are highly luminescent (33\% QY for DT/MUO-capped nanocrystals in methanol and $23 \%$ QY for DT/MUA-capped nanocrystals in water). These results obtained in the previous and the present work imply that the optical properties of the ligand stabilized nanocrystals significantly depend on the molecules used as protective regents and the process used for surface functionalization, both of which can result in formation of surface trap sites and/or compensate surface defects during the surface chemical modification process. Nevertheless, the process reported herein is a powerful method for producing highly luminescent, surface-functionalized nanocrystals that can be handled as solid macromolecules using a straightforward procedure. These stable nanocrystals may be useful for a wide range of optoelectronic and sensing devices.

\section{CONCLUSION}

In conclusion, we have demonstrated that thiol-capped, highly luminescent CdTe nanocrystals with QYs as high as $50 \%$ can be synthesized using a simple surface modification approach. The process fully relies on the surface attachment of thiol molecules to the nanocrystal surface, altering the initially highly defective (silent) nanocrystals to less defective (fluorescent) nanocrystals. The strong binding affinity of mercapto groups for the
$\mathrm{Cd}$ atoms enables the resulting purified nanocrystals to be stable for a long time. Thus, these crystals can be stored as solid powders without any loss in the structural or optical characteristics. One of the advantages of the process is that the surface functionality of the nanocrystals can be further tailored using various molecules through place exchange reaction, by which mixed monolayer-functionalized nanocrystals with solubility in various solvents may be synthesized. Moreover, these nanocrystals with diverse surface properties can now be investigated for their potential use as building blocks in functional materials, such as thin film light emitters and biological labels.

\section{REFERENCES}

[1] A. P. Alivisatos, J. Phys. Chem., 100, 13226 (1996)

[2] S. Coe, W.-K. Woo, M. G. Bawendi, V. Bulovic, Nature, 420, 800 (2000)

[3] V. I. Klimov, A. A. Mikhailovsky, S. Xu, A. Malko, J. A. Hollingsworth, C. A. Leatherdale, H.-J. Eisler, M. G. Bawendi, Science, 290, 314 (2000)

[4] M. Han, X. Gao, J. Z. Su, S. Nie, Nat. Biothechnol., 19, 631 (2001)

[5] M. A. Hines, P. Guyot-Sionnest, J. Phys. Chem., 100 468 (1996)

[6] D. V. Talapin, A. L. Rogach, A. Kornowski, M. Haase, H. Weller, Nano Lett. 1, 207 (2001)

[7] D. V. Talapin, R. Koeppe, S. Götzinger, A. Kornowski, J. M. Lupton, A. L. Rogach, O. Benson, J. Feldmann, H. Weller, Nano Lett., 3, 1677 (2003)

[8] J. J. Li, Y. A. Wang, W. Guo, J. C. Keay, T. D. Mishima, M. B. Johnson, X. Peng, J. Am. Chem. Soc., 125, 12567 (2003)

[9] P. Reiss, J. Bleuse, A. Pron, Nano Lett., 2, 781 (2002)

[10] H. Bao, Y. Gong, Z. Li, M. Gao, Chem. Mater., 16, 3853 (2004)

[11] S. F. Wuister, I. Swart, F. van Driel, S. G. Hickey, C. de Mello Donegá, Nano Lett., 3, 503 (2003)

[12] S. F. Wuister, C. de Mello Donegá, A. Meijerink, J. Phys. Chem. B, 108, 17393 (2004)

[13] G. Kalyuzhny, R. W. Murray, J. Phys. Chem. B, 109, 7012 (2005)

[14] H. T. Uyeda, I. L. Medintz, J. K. Jaiswal, S. M. Simon, H. Mattoussi, J. Am. Chem. Soc., 127, 3870 (2005)

[15] T. Tsuruoka, R. Takahashi, T. Nakamura, M. Fujii, K. Akamatsu, H. Nawafune, Chem. Commun., 1641 (2008)

[16] W. W. Yu, L. Qu, W. Guo, X. Peng, Chem. Mater., 15, 2854 (2003)

[17] Y. Nonoguchi, T. Nakashima, T. Kawai, J. Phys. Chem. C, 113, 11464 (2009)

[18] N. Gaponik, D. V. Talapin, A. L. Rogach, A. Eychmuller, H. Weller, Nano Lett., 2, 803 (2002)

(Received December 24, 2010; Accepted February 26, 2011) 